\title{
Novel detectors for traceable THz power measurements
}

\author{
Ralf Müller • Werner Bohmeyer • Mathias Kehrt • \\ Karsten Lange $\cdot$ Christian Monte $\cdot$ Andreas Steiger
}

Received: 5 November 2013 / Accepted: 13 March 2014 /

Published online: 2 April 2014

(C) The Author(s) 2014. This article is published with open access at Springerlink.com

\begin{abstract}
Several novel types of detectors for the measurement of electromagnetic radiation in the $\mathrm{THz}$ spectral range are described. Firstly, detectors based on pyroelectric foil coated with different absorbers have been developed focusing on the following features: high accuracy due to well-characterized absorption, high sensitivity, large area absorbers and frequency and polarization independence. A three-dimensional design with five absorptions gave an overall absorption of more than $98 \%$. Secondly, detectors based on pyroelectric foils with thin metal layers were realized. An absorption of $50 \%$ can be obtained if the thickness of the layers is carefully adjusted. According to electromagnetic theory this degree of absorption is independent of the polarization and frequency of the radiation in a wide range from at least $20 \mathrm{GHz}$ to $5 \mathrm{THz}$. The third type of detector is based on a new type of volume absorber with a polished front surface and a gold-coated back side. It is the absorber of choice of the standard power detector for disseminating the spectral power responsivity scale. This standard detector allows the application of a physical model to calculate its spectral responsivity in the range from $1 \mathrm{THz}$ to $5 \mathrm{THz}$ if the detector has been calibrated at one single frequency. Finally, a $\mathrm{THz}$ detector calibration facility was set up and is now in operation at PTB to calibrate detectors from customers with an uncertainty as low as $1.7 \%$.
\end{abstract}

Keywords Far infrared detectors $\cdot$ Terahertz detectors $\cdot$ Radiometry $\cdot$ Metrological instrumentation $\cdot$ Infrared and far infrared lasers $\cdot$ Optics

\section{Introduction}

Besides frequency, radiant power is the most important physical quantity for characterizing optical radiation sources. There are two main types of detectors for the measurement of radiant power, either thermal or photonic devices. Photonic detectors are based on the photoelectric

R. Müller $\cdot$ M. Kehrt $\cdot$ C. Monte $\cdot$ A. Steiger $(\bowtie)$

Physikalisch-Technische Bundesanstalt (PTB), Abbestr. 2-12, 10587 Berlin, Germany

e-mail: Andreas.Steiger@ptb.de

W. Bohmeyer $\cdot \mathrm{K}$. Lange

Sensor- und Lasertechnik (SLT), Schulstr. 15, 15366 Neuenhagen, Germany

W. Bohmeyer

e-mail: sevice@pyrosensor.de 
effect and are predominantly used at higher frequencies in the visible, ultraviolet and X-ray spectral range. In contrast, thermal detectors are mainly used at longer wavelengths, i.e. in the infrared up to the THz spectral range and at higher power levels as well. They absorb the incident radiation and simply heat an absorbing material. The heat is then detected by temperature-dependent phenomena such as the temperature-dependent conductivity, the thermo- or pyroelectric effect.

All thermal effects depend only on the absorbed radiation power and are independent of photon energy. Therefore, thermal detectors are generally broadband measuring instruments. Pyroelectric detectors are in this class of thermal detectors. Their responsivity shows in principle no wavelength dependency of the incoming radiation provided their absorption does not depend on wavelength. Most detectors offered meet this condition well in the visible and near infrared range. With increasing wavelength, however, it becomes more and more difficult to realize a wavelength-independent absorption [1-4]. This is because most of the well-known absorbers - even those containing carbon nanotubes (CNT) - exhibit an increasing transmittance with increasing wavelength [5]. To overcome this limitation, PTB in cooperation with SLT has developed several types of novel detectors suitable for application in the THz range.

\section{Detector development}

As a result of the increasing transmittance of the absorber material with a longer wavelength, the incoming radiation is only partly absorbed on its way through the absorber along to the back side of the detector where the metallic electrode of the detector material is located. This metallic layer acts as a reflector for the far infrared (FIR) radiation. The transmitted part of the incoming radiation passes again through the absorber. For thin absorbers, even two transitions are not enough for a total, i.e. wavelength-independent, absorption. The advantage of $\mathrm{THz}$ radiation to penetrate a large variety of dielectric materials acts here as a disadvantage. In principle, the thickness of the absorber can be increased but the rise and decay times grow so that the sensitivity is drastically reduced. For thermal power meters, the thickness of the absorber can be increased leading to higher absorbance and to time constants in the order of seconds. For energy meters, a rise time in the ms region is desired and thin absorbers are therefore necessary.

Much research work has been done in the last few years in this field [6]. Different techniques are known in the microwave and FIR region to increase the absorbance by using homogeneous electrically conducting coatings or by generating conductive absorbing structures. The dimensions of structures produced by photolithography must be small compared to the wavelength. Then a broadband absorption can be reached using a structured coating [7]. On the contrary, this method can also be used to reach very high absorption but only for a selected wavelength [8]. As an unfavourable result the absorption strongly drops down at neighbouring wavelengths. The challenge to make a $\mathrm{THz}$ detector which can be easily calibrated is not to get an absorbance of $100 \%$ but a wavelength-independent absorbance. A higher degree of absorption increases the responsivity but is not an imperative condition for the calibration of the detector.

The absorbance of different organic black coatings with and without CNT, whose layer thickness is in the range of $10 \mu \mathrm{m}$ to $80 \mu \mathrm{m}$, was investigated. An FIR transparent carrier was used for these tests to measure both the transmission and the reflection (see Fig. 1). The best result achieved is an absorbance of more than $50 \%$ at $500 \mu \mathrm{m}$ with a decreasing tendency towards longer wavelengths. Furthermore, the reflection increases up to $40 \%$ at $500 \mu \mathrm{m}$. This coating is not acceptable for high-quality $\mathrm{THz}$ detectors. 


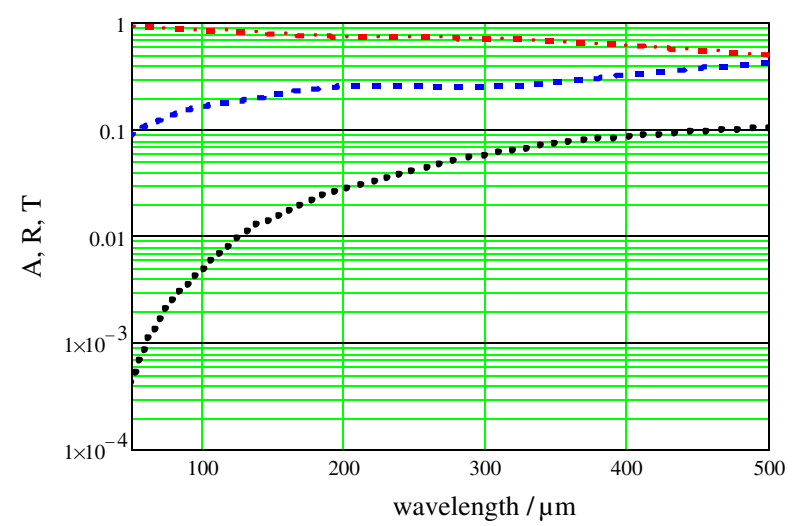

Fig. 1 Spectral properties of an organic black coating with CNT, thickness about $80 \mu \mathrm{m}$, on a THz transparent carrier. The absorbance (red upper line) is calculated from the measured reflectance (blue dashed line) and transmittance (black dotted line).

\subsection{Trap detector}

Another way to increase the absorption is by implementing a special trap detector design. The advantage of a three-element trap detector is the fact that the incoming radiation interacts up to five times with the detector surface. The total absorbance $A$ of this set up is $A=1-R^{5}$, where $R$ is the reflectance for a single interaction with the detector. The three identical detectors are arranged in such a way that the radiation strikes the first and second detectors under an angle of $45^{\circ}$, while the third one acts as a reflector. The three detectors have a $3 \mathrm{D}$ arrangement to achieve a polarization-independent responsivity. The above mentioned coating (Fig. 1) is used for the trap detector. Its setup is shown in Fig. 2.

A nearly wavelength-independent responsivity $( \pm 3 \%)$ can be realized by the trap arrangement if the absorbance of a single interaction is at least $50 \%$ (Fig. 3). It must be guaranteed for all wavelengths that all four possible reflections strike the surface of the next detector. In order

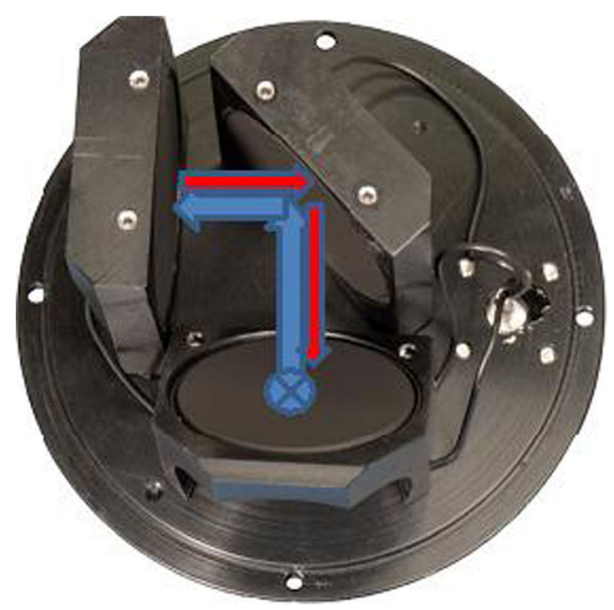

Fig. 2 Photo of a THz trap detector without cover. The arrangement of the three detectors is clearly visible. The radiation path originating from the observer and reflected by the three surfaces is depicted by arrows. The maximum accepted beam diameter of this detector design is $25 \mathrm{~mm}$. 


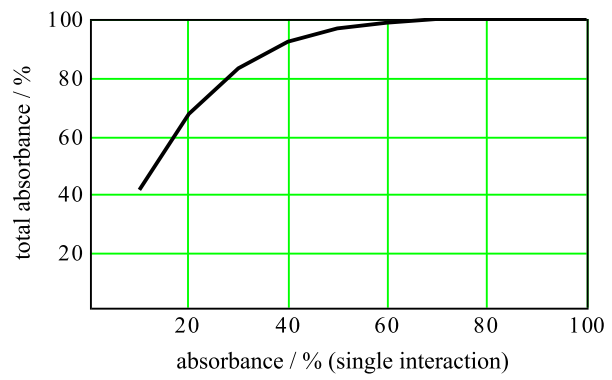

Fig. 3 The total absorbance for a three-element trap in relation to the absorbance for a single interaction. A single absorbance of $50 \%$ results in a total absorbance of $97 \%$.

to meet this condition the allowed angle of acceptance is small. This small angle of acceptance for incoming radiation can be increased by using concave detectors [9].

Well-fabricated trap detectors exhibit a high wavelength-independent responsivity and a good spatial homogeneity as well. The latter is shown for two orthogonal scans over the detector in Fig. 4. Due to its high absorption the detector described in this section shows about twice the sensitivity of the detector described in the next Section.

\subsection{Novel single-element detectors}

Another interesting concept developed to realize a flat absorption in the FIR was published as long ago as 1934 [10]. The concept is based on the solution of Maxwell's equations for conductive coatings with a thickness that is smaller than the wavelength and the penetration depth of the optical radiation. By matching the sheet resistance of the coating to half of the vacuum impedance, a broadband absorption of $50 \%$ can be realized. This concept is, for example, realized in a commercially available photoacoustic "Absolute Terahertz Power/ Energy Meter" from Thomas Keating Ltd. by using a dielectric carrier foil and a thin metal coating with a matched area resistance. Here the disadvantages are the necessity of a thick optical window and a large time constant restricting repetition rates to lower than $50 \mathrm{~Hz}$.

But the concept can also be used advantageously for a thin film pyroelectric detector: its metal electrodes can act as a THz radiation absorber by optimizing their area resistance on both

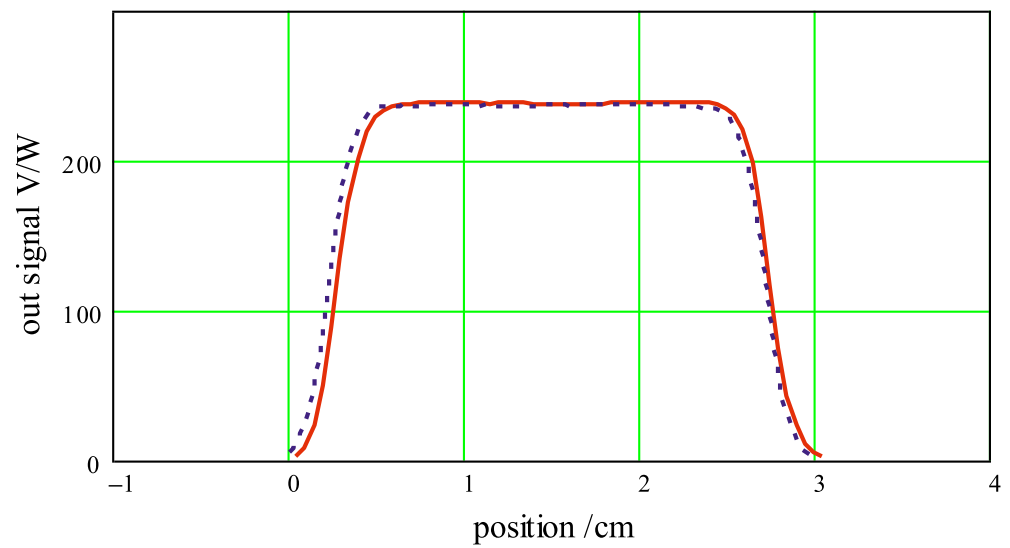

Fig. 4 Scan profiles across a trap detector in two orthogonal directions at $2.52 \mathrm{THz}$. 


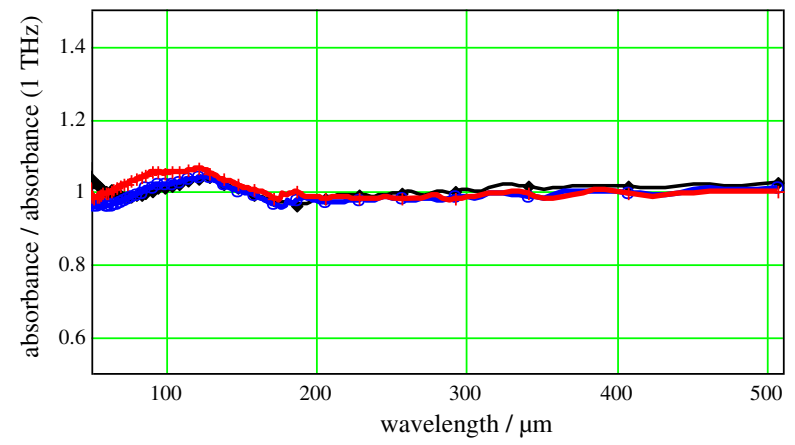

Fig. 5 Wavelength-independent absorbance calculated from measured reflectance and transmittance for three samples. The measured values are normalized to the absorbance at $300 \mu \mathrm{m}(1 \mathrm{THz})$.

sides to reach a nominal absorbance of $50 \%$ without any additional coating. Figure 5 shows the normalized absorbance for three randomly selected samples with such a type of coating.

Figure 5 demonstrates an excellent reproducibility of the thin metal coating of the three samples. The achieved tolerances in production guarantee a good quality. Only for wavelengths shorter than $150 \mu \mathrm{m}$ do absorption bands of the pyroelectric foil lead to a slightly increased absorption. Between $150 \mu \mathrm{m}$ and $600 \mu \mathrm{m}$ the maximum deviation is less than $4 \%$.

This old knowledge was applied to develop a new type of pyroelectric $\mathrm{THz}$ detector. The necessary area resistances can be as high as $1000 \Omega$. Advanced technology is essential for making reliable and large area coatings. In this context the following issues have attracted attention:

- Diffraction limits to focus $\mathrm{THz}$ radiation into very small detectors. Consequently, the detectors were designed to have active areas between $10 \mathrm{~mm}$ and $30 \mathrm{~mm}$ in diameter.

- In order to achieve a fast and high response, the heat capacity of the absorber should be as small as possible and the thermal conductivity of the absorber should be as high as possible. Both are fulfilled using thin metallic coatings.

- The responsivity increases with the decreasing thickness of the pyroelectric detector material but technological problems arise for materials thinner than $10 \mu \mathrm{m}$.

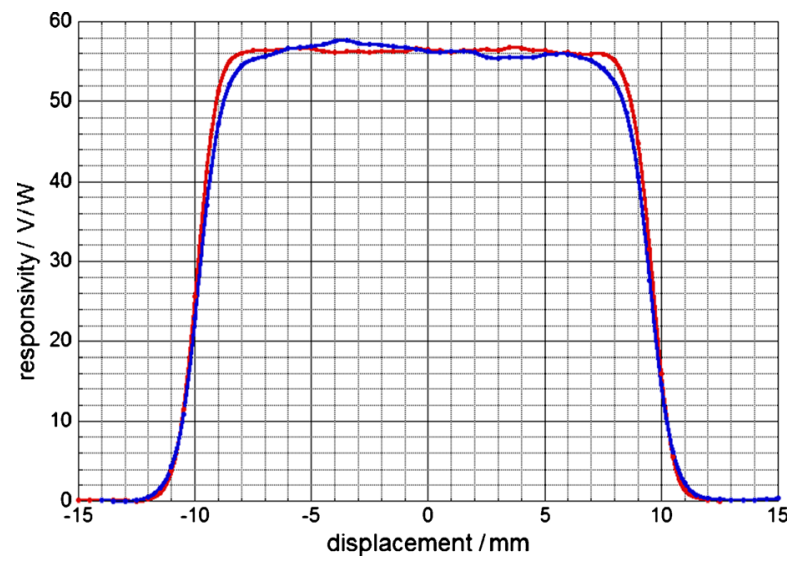

Fig. 6 Responsivity scan profiles across an SLT's THz20 detector in two orthogonal directions at 2.52 THz. 


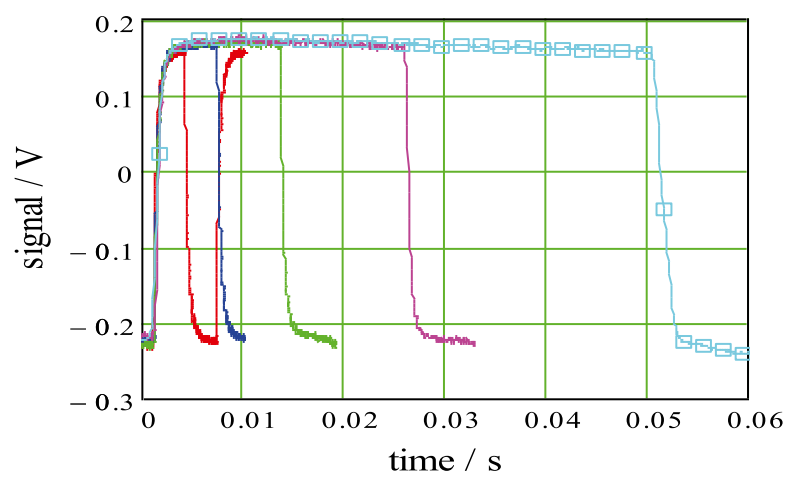

Fig. 7 Output signal of the transimpedance amplifier of a THz20 detector combined with a chopper at modulation frequencies of $10 \mathrm{~Hz}$ (light blue), $20 \mathrm{~Hz}, 40 \mathrm{~Hz}, 80 \mathrm{~Hz}$ and $160 \mathrm{~Hz}$ (red). Due to the almost instantaneous rise and decay of the current signal and the large thermal time constant of about 2 s, nearly rectangular pulse shapes are depicted at all modulation frequencies.

- The electrical capacity increases for thin and large area detectors which is a disadvantage for energy meters. In most cases the pyroelectric detectors are used in current mode with a transimpedance amplifier for power measurement. In this case, the increased capacity is less critical.

- The dimensioning of the absorber coating is more flexible if the dielectric constant of the detector material is low.

- In order to avoid air movement and noise, a chopper frequency in the range from $30 \mathrm{~Hz}$ to $50 \mathrm{~Hz}$ is often desired. A correct measurement of these pulses requires a thermal time constant, i.e. the time for reaching temperature equilibrium with the environment, to be in the order of one second. Then the output signal will decrease less than $2 \%$ in $20 \mathrm{~ms}$.

The following invention was made to meet all of the above-mentioned demands. An approximately $10 \mu \mathrm{m}$ thick pyroelectric active polyvinylidene difluoride (PVDF) foil acts as the detector material. It is coated on both sides with a thin metallic absorber film. The foil is clamped between two rings which are also used to electrically connect the read-out. The main heat loss mechanism is convection with a thermal time constant of about one second. The detectors are windowless to achieve a wavelength-independent responsivity. In this case any movement of air or acoustic noise produce a noticeable signal and should be avoided.

Figure 6 shows scan profiles across a THz20 detector at $2.52 \mathrm{THz}$. An outstanding attribute of all detectors with metallic absorbers is their perfect spatial homogeneity.

A wavelength-independent absorbance of $50 \%$ can be realized if the area resistance $R_{\mathrm{r}}$ of the back side coating of the detector and the area resistance $R_{\mathrm{f}}$ of the front side coating meet the condition $1 / R_{\mathrm{r}}+1 / R_{\mathrm{f}}=2 / \mathrm{Z}_{\mathrm{o}}$ where $\mathrm{Z}_{0}$ is the impedance of the vacuum. In this case there is $25 \%$ reflectance and $25 \%$ transmittance.

Typical current sensitivities for PVDF are $0.5 \mu \mathrm{A} / \mathrm{W}$. Combined with a transimpedance preamplifier with an amplification of $10^{9} \mathrm{~V} / \mathrm{A}$, a responsivity of up to $500 \mathrm{~V} / \mathrm{W}$ can be reached [11]. The noise equivalent power is below $10 \mu \mathrm{W}$. This enables a precise determination of the $\mathrm{THz}$ power responsivity of these pyroelectric detectors because the $\mathrm{THz}$ laser emits more than $1 \mathrm{~mW}$ as described in Section 3. The fast time response of the output signal of a THz20 is depicted in Fig. 7. It reveals a rise and decay time less than $1.5 \mathrm{~ms}$. Thus repetition rates higher than $100 \mathrm{~Hz}$ are measureable. 


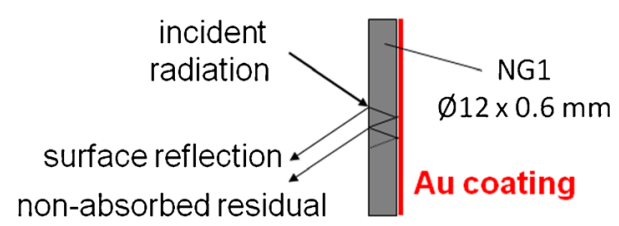

Fig. 8 Schematic diagram of the radiation absorption process shown by a sectional drawing of the gold-coated NG1 disc.

\subsection{A novel detector as a power measurement standard}

In contrast to the detectors described in the previous sections a well-suited standard detector for power measurement does not need to have a flat spectral responsivity in the THz range but rather one which is well known. This is achieved by two steps:

A detector is needed where the frequency dependence of its spectral responsivity can be derived from a physical model. The physical model described in this section together with the characterization of the spectral reflectance and transmittance result in a known spectral absorbance of the detector.

As the second step, this well-characterized detector is then calibrated at one single frequency that may or may not be within the $\mathrm{THz}$ range. Through this single point calibration its spectral absorbance is transformed into a spectral power responsivity which is traceable to the International System of Units (SI) if the single point calibration is also traceable.

These two steps yield a detector that can be used as a standard detector in the THz range.

The standard detector is a modified commercial laser power meter. It is a highly sensitive thermopile detector, model 3A-P from Ophir Optronics Ltd., which uses a $0.6 \mathrm{~mm}$ thin disc of $12 \mathrm{~mm}$ diameter made of NG1 neutral density filter glass from SCHOTT AG as a volume absorber. In contrast to the commercial model an optically polished plane surface is used instead of a matt surface. In addition, the back side of the NG1 disc is coated with a thin gold layer which acts as a mirror for the residually transmitted radiation in order to achieve a longer absorption path. This is depicted in Fig. 8. Both modifications make a simple physical model of the radiation losses applicable [12].

The model describes the frequency-depending absorbance $A(\nu)$ of the new standard detector in the THz region but also in the visible spectral region. Due to the optical quality of the NG1 material and the polishing of its surfaces, radiation scattering or diffuse reflection can be neglected. Only specular reflection has to be taken into account for the THz radiation which is not absorbed by the NG1 disc. The major losses originate from the first reflection when incident radiation impinges on its surface. Most of the penetrating radiation is absorbed by the NG1 material. However, at frequencies below $1 \mathrm{THz}$ NG1 becomes transparent. But due to the gold-coated back side all residually transmitted radiation is reflected and is partially absorbed on its way back to the first surface. Because of the lower refractive index of the air with respect to the NG1, only a minor part is transmitted out of the NG1 and most of the residual $\mathrm{THz}$ radiation is reflected again towards the reflective back side, is then again partially absorbed, and so on. Because the front and back side of the NG1 disc are parallel the nonabsorbed residual radiation has the same direction as the first surface reflection as indicted by a parallel slim line in Fig. 8. The resulting interference of the parallel beams is of minor importance as long as there is a noticeable absorption by the NG1 disc which occurs for $\mathrm{THz}$ radiation above $0.7 \mathrm{THz}$.

In any case, radiation which is not absorbed inside the NG1 disc is reemitted in the same direction as the front surface reflection. This is the only significant optical loss that has to be 
taken into account. The reflectance $R(\nu)$ is determined by a measurement of the ratio of the reflected to the incident radiation at any frequency $\nu$. The absorbance of the NG1 volume absorber of the standard detector is given according to the model:

$$
A(\nu)=1-R(\nu) \text {. }
$$

As mentioned above this detector has to be calibrated at a single frequency $\nu_{0}$, i.e. its power responsivity $s\left(\nu_{0}\right)$ has to be determined. Then the power responsivity $s(\nu)$ at a frequency $\nu$ can be calculated by taking the ratio of absorbance into account:

$$
s(\nu)=s\left(\nu_{0}\right) \cdot \frac{A(\nu)}{A\left(\nu_{0}\right)}=s(2.52 \mathrm{THz}) \cdot \frac{1-R(\nu)}{1-R(2.52 \mathrm{THz})} .
$$

Equation (2) holds for all frequencies $\mathrm{v}_{0}$ where the power responsivity is known and for all frequencies $\nu$ where the physical model is applicable, i.e. the frequencies where reflectance and transmittance of the absorber of the standard detector are known. These frequencies may also be within the visible spectral range.

The visible range is used for the single point calibration because the reflectance of NG1 could be measured with a HeNe laser with high accuracy. The result is $R_{\mathrm{HeNe}}=(4.38 \pm 0.03) \%$. This low value is consistent with the refractive index of $n=1.52$ in the visible spectral range published in the datasheet of NG1 of the manufacturer.

Finally, the radiant power responsivity $s_{\mathrm{HeNe}}$ of the standard detector is determined by means of a HeNe laser. Thanks to the optical quality of NG1 and the resulting good homogeneity within its aperture of $12 \mathrm{~mm}$ diameter, $s_{\mathrm{HeNe}}$ has an uncertainty as low as $0.2 \%$ and is traceable to SI units because the calibration is performed at the Laser Radiometry Working Group of PTB responsible for the dissemination of radiometric units for laser radiometry in the optical wavelength range and adjacent spectral regions [13, 14].

Equation (2) can thus be rewritten as follows:

$$
s(\nu)=\frac{s_{\mathrm{HeNe}}}{0.9562} \cdot[1-R(\nu)]
$$

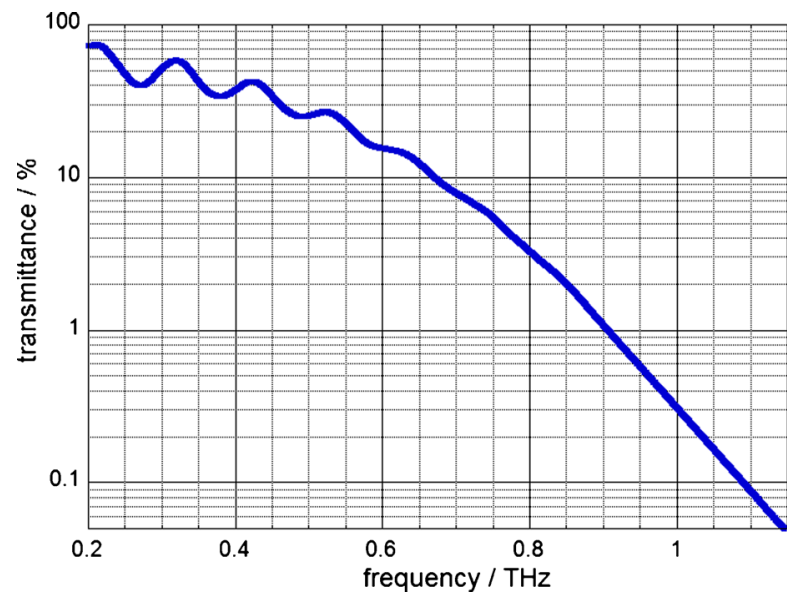

Fig. 9 Spectral THz transmittance of a $0.57 \mathrm{~mm}$ thick NG1 sample. The signal modulation at THz frequencies below $0.8 \mathrm{THz}$ is due to interference caused by standing waves inside the plane parallel sample. 


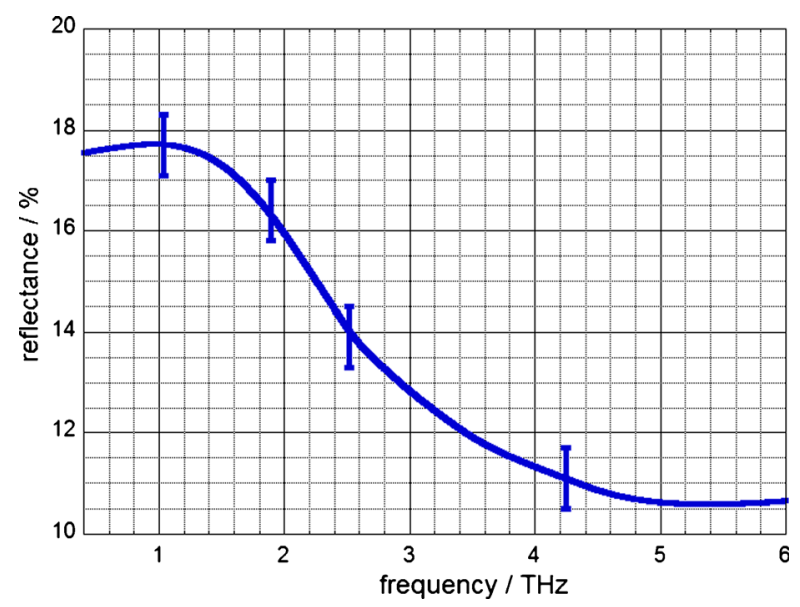

Fig. 10 Spectral THz reflectance of a $0.57 \mathrm{~mm}$ thick NG1 sample. The typical standard uncertainty is shown for some laser lines.

The quantities in Equation (3) can be determined with low uncertainty because $s_{\mathrm{HeNe}}$ and $R_{\mathrm{HeNe}}$ are based on accurate measurements in the visible spectral range.

To use the physical model the spectral transmittance and reflectance in the THz range have to be measured. The transmittance in the far infrared spectral range was measured in this work using a Fourier transform infrared (FT-IR) spectrometer, namely a VERTEX 80v vacuum FTIR spectrometer from the Bruker Corporation. The result for a $0.57 \mathrm{~mm}$ thick sample of the same batch as the gold-coated NG1 disc absorber of the standard detector is shown in Fig. 9.

The reflectance of an NG1 sample with the gold coating on its back side has to be measured as well (see Fig. 10). However, the diameter of $12 \mathrm{~mm}$ of the absorber disc is too small to measure the reflected signal without any losses due to clipping and diffraction of radiation at the long wavelengths of the $\mathrm{THz}$ spectral range. Therefore, NG1 discs of $40 \mathrm{~mm}$ diameter were ordered together with the $12 \mathrm{~mm} \mathrm{NG1}$ absorber discs and coated with gold in the same process run. The gold-coated NG1 sample with $40 \mathrm{~mm}$ in diameter has the same thickness of $0.57 \mathrm{~mm}$ and will therefore have the same reflection as the gold-coated NG1 absorber of the new standard detector.

The reflectance of the 40 diameter NG1 sample was measured in the spectral range from $1 \mathrm{THz}$ to $5 \mathrm{THz}$ by two independent methods. In addition to measurements with the FT-IR spectrometer, different emission lines of a molecular gas laser were used. By using the $\mathrm{THz}$ laser the absolute measurement uncertainty is not more than $0.5 \%$. The measurement by means of the FT-IR spectrometer reveals consistent reflectance values with an uncertainty larger than $1 \%$ (Fig. 10).

Within the spectral range of interest from $1 \mathrm{THz}$ to $5 \mathrm{THz}$ the losses due to the reflectance $R(\nu)$ of the NG1 sample vary between $18 \%$ and $10 \%$. According to Equation (1), the corresponding spectral absorbance $A(\nu)$ varies only between $82 \%$ and $90 \%$. An absolute measurement uncertainty of $0.5 \%$ of $R(\nu)$ yields a relative uncertainty of $A(\nu)$ well below $1 \%$ for any frequency $\nu$ in the THz spectral range. The combined uncertainty of the THz power responsivity scale $s(\nu)$ is therefore $1.2 \%$ and is mainly caused by the uncertainty of the reflectance $R(\nu)$ at $\mathrm{THz}$ frequencies.

This modified detector is now used as a standard detector for power measurement and is integrated into a facility for detector calibration at PTB. 


\section{Detector calibration facility}

Detector-based radiometry with laser radiation [15] and the standard detector described in the previous section was chosen as the optical method for the dedicated $\mathrm{THz}$ power responsivity calibration facility of PTB. This method with a laser as a time-stable monochromatic radiation source is well established for visible and infrared radiation but also suited to the long wavelengths of $\mathrm{THz}$ radiation. The main advantages of laser-based calibration in the $\mathrm{THz}$ range are: high spectral purity, low stray light and the lowest possible beam divergence. The latter properties facilitate the focusing of the radiation in order to underfill the detector aperture which is the crucial part of absolute THz power measurements [16].

Figure 11 shows the configuration for laser-based spectral responsivity measurement at the $\mathrm{THz}$ detector calibration facility. The core instrument is an optically pumped $\mathrm{THz}$ laser. It is a far-infrared molecular gas laser which was commercially available until 2010 [17]. The laser system delivers an output power of multiple milliwatt continuous wave (cw) at frequencies between $1 \mathrm{THz}$ and $5 \mathrm{THz}$ at a variety of rotational emission lines of selected molecular gases at low pressures in the range of $10 \mathrm{~Pa}$ to $100 \mathrm{~Pa}$. For example, operated with methanol as the laser medium and with $\mathrm{CO}_{2}$ pump radiation at $9.7 \mu \mathrm{m}$, the methanol molecules lase between the $\mathrm{J}=16$ and $\mathrm{J}=15$ rotational levels emitting radiation at $2.52 \mathrm{THz}$ which corresponds to a wavelength of $119 \mu \mathrm{m}$. This is the same wavelength which was used for the first calibration experiment at PTB based on a quantum cascade laser [18].

Due to diffraction of the long wavelengths, the $\mathrm{THz}$ laser beam has a large divergence which requires a diameter of $75 \mathrm{~mm}$ for the first focusing mirror at a $178 \mathrm{~mm}$ distance to the output-coupling hole of the THz laser. A 90 degree off-axis parabolic mirror (OAPM) with a $178 \mathrm{~mm}$ focal length is used to produce a collimated beam which is focused by another OAPM into the aperture of an iris diaphragm acting as a spatial filter. The diameter and position of the iris diaphragm are adjusted to achieve a Gaussian-like beam profile (see Fig. 12). The diaphragm is tilted off normal incidence by 10 degrees in order to avoid standing waves by any reflected radiation of its blade.

A chopper is placed at a short distance behind the diaphragm. The gold-plated chopper blade has two slots and a diameter of $200 \mathrm{~mm}$. The large chopper wheel and the small beam diameter at a short distance behind the focus result in a symmetric on-off power modulation with a small $0.5 \%$ ratio of rise time to a modulation period at typical modulation frequencies in the range of $10 \mathrm{~Hz}$ to $50 \mathrm{~Hz}$. As the chopper blade is coated with a gold layer and oriented at 45 degrees, the reflected $\mathrm{THz}$ beam can be used to monitor the power variation during the calibration process. An aspheric plane-convex Tsurupica lens with a $30 \mathrm{~mm}$ clear aperture and a $50 \mathrm{~mm}$ focal length is used to focus the reflected beam without clipping any $\mathrm{THz}$ radiation onto the absorbing surface (12 $\mathrm{mm}$ diameter) of the monitor detector. The transmitted beam is

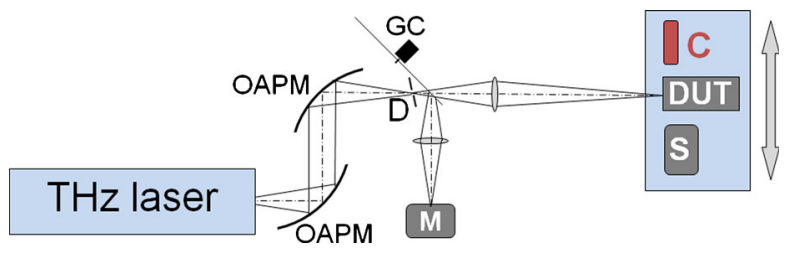

Fig. 11 Measurement setup of the THz calibration facility. The radiation emitted by the THz laser is imaged by two off-axis parabolic mirrors (OAPM) onto the aperture of an iris diaphragm (D). The transmitted radiation is focused by a lens into the detector under test (DUT), into the standard detector (S) or into a THz camera (C) which are all mounted on top of a linear translation stage. The reflected radiation from the surface of a gold-plated chopper blade (GC) is focused into a monitor detector (M) by another lens. 


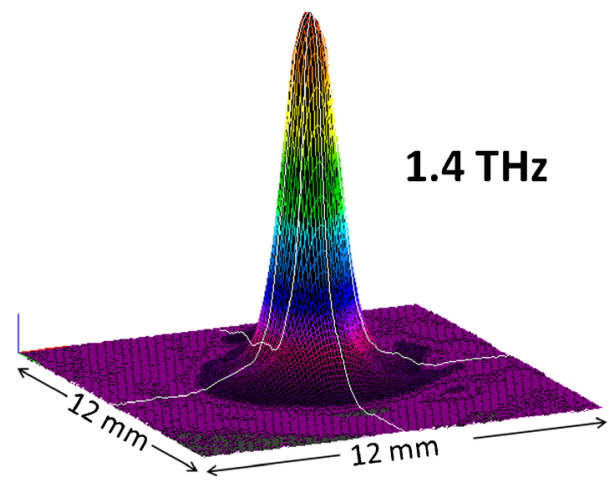

Fig. $123 \mathrm{D}$ plot of a typical focal beam profile of the molecular gas laser at $1.4 \mathrm{THz}$. The sensitive area of the $\mathrm{THz}$ camera is $12 \mathrm{~mm}$ x $12 \mathrm{~mm}$.

focused by another Tsurupica lens with a $100 \mathrm{~mm}$ focal length onto the absorbing surface of the detector under test (DUT).

The detector at the focus position is mounted on top of a horizontal translation stage together with the standard detector and a $\mathrm{THz}$ camera. The displacement range of $400 \mathrm{~mm}$ of this translation stage is used to position these devices one after another into the focus of the $\mathrm{THz}$ laser beam and to measure the spatial variation of the sensitivity of the DUT in the horizontal direction. The mechanical design includes two lifting tables with a $30 \mathrm{~mm}$ travel range which are used to vary the vertical position of these devices.

The calibration procedure is as follows: First the focal beam profile is recorded by the $\mathrm{THz}$ camera (see Fig. 11). Then the power is measured by the standard detector. The signal of the monitor detector is recorded at the same time. Finally, the DUT is moved into the focus of the $\mathrm{THz}$ beam to the same position as the standard detector and the reading of the DUT and the signal of the monitor detector are stored. The responsivity of the DUT is calculated as the ratio of its reading to the power measured with the standard detector. The signal of the monitor detector is used to correct any temporal drift of the laser output power during these measurements.

\section{Conclusion}

The detector calibration facility in combination with the standard detector enables PTB as the national metrology institute of Germany to offer the accurate calibration of suitable $\mathrm{THz}$ detectors as an official service which is unique in the world. The standard uncertainty of the $\mathrm{THz}$ radiant power scale is $1.2 \%$ and the uncertainty of power responsivity calibrations of detectors can be as low as $1.7 \%$ in the spectral range from $1 \mathrm{THz}$ to $5 \mathrm{THz}$.

This low uncertainty can be achieved for calibrations of single absorber or trap detectors described in Section 2.1. These detectors are now available for a broad frequency range in the $\mathrm{THz}$ region and can be used even far beyond the calibration range from $1 \mathrm{THz}$ to $5 \mathrm{THz}$. For instance, the lower end of operation range of a metallic-coated thin film pyroelectric detector is below $20 \mathrm{GHz}$.

Therefore, these detectors pave the way for the accurate power measurement of broadband $\mathrm{THz}$ sources and bridge the gap towards the $\mathrm{GHz}$ range. 
Acknowledgment The THz detector development of Section 2.1 was performed in a close cooperation project financially supported by the Investitionsbank Berlin (IBB) and the Investitionsbank Brandenburg (ILB) cofunded by the European Regional Development Fund.

Other parts of this work have been supported by the ongoing joint research project "THz Security" with the number NEW07 within the European Metrology Research Programme (EMRP). The EMRP is jointly funded by the EMRP participating countries within EURAMET and the European Union.

Open Access This article is distributed under the terms of the Creative Commons Attribution License which permits any use, distribution, and reproduction in any medium, provided the original author(s) and the source are credited.

\section{References}

1. D. B. Betts, F. J. J. Clarks, L. J. Cox, J. A. Larkin, J. Phys. E: Sci. Instrum. 18, 689 (1985).

2. S. Ungar, J. Mangin, M. Lutz, G. Jeandel, B. Wyncke, SPIE 1157 Infrared Technology XV, 369 (1989)

3. Hu Tao, C. M. Bingham, A. C. Strikwerda, D. Pilon, D. Shreckenhamer, N. I. Landy, K. Fan, X. Zhang, W. J. Padilla, R. D. Averitt, Phys. Rev. B 78, 241103(R) (2008)

4. A. K. Azad, D. Y. Shchegolkov, H.-T. Chen, A. Taylor, E. Smirnova, J. O'Hara, in Conference on Lasers and Electro-Optics (CLEO), OSA Technical Digest (CD) paper CTuBB (2010)

5. J. H. Lehman, B. Lee, E. N. Grossman, Appl. Optics 50, 4099 (2011)

6. Z. Popović, E. N. Grossman, IEEE Transact. THz Sci. Technol. 1, 133 (2011)

7. M. Kehrt, J. Beyer, C. Monte, J. Hollandt, 38th International Conference on Infrared, Millimeter and Terahertz Waves (IRMMW-THz), paper Mo8-4 (2013)

8. S. A. Kuznetsov, M. A. Astafyev, A. V. Arzhannov, M. K. Thumm, 38th International Conference on Infrared, Millimeter and Terahertz Waves (IRMMW-THz), paper Th2-2 (2013)

9. W. Bohmeyer, K. Lange, A. Steiger, R. Müller, Patent DE102012008477

10. W. Woltersdorff, Zeitschrift für Physik 91, 230 (1934)

11. www.pyrosensor.de/THZ_e.pdf

12. A. Steiger, M. Kehrt, C. Monte, R. Müller, Opt. Express 21, 14466 (2013)

13. BIPM key comparison database (February 2013), Calibration and measurement capabilities, photometry and radiometry, Germany, PTB, second item on page 4 of http://kcdb.bipm.org/appendixC/PR/DE/PR_DE.pdf

14. X. Li, T. Scott, C. Cromer, D. Keenan, F. Brandt, K. Möstl, Metrologia 37, 445 (2000)

15. L. P. Boivin, Optical Radiometry, A. C. Parr, R. U. Datla, J. L. Gardner, eds. (Elsevier 2005), pp. 97

16. A. Steiger, B. Gutschwager, M. Kehrt, C. Monte, R. Müller, J. Hollandt, Opt. Express 18, 21804 (2010)

17. E. R. Mueller, www.coherent.com/downloads/OpticallyPumpedLaser.pdf

18. L. Werner, H.-W. Hübers, P. Meindl, R. Müller, H. Richter, A. Steiger, Metrologia 46, S160 (2009) 